\title{
Cell Contour Tracking and Data Synchronization for Real-Time, High-Accuracy Micropipette Aspiration
}

\author{
Xinyu Liu, Student Member, IEEE, Yifei Wang, Student Member, IEEE, and Yu Sun, Senior Member, IEEE
}

\begin{abstract}
This paper presents an automated cell contour visual measurement technique and a data synchronization mechanism for real-time, high-accuracy mechanical characterization of individual cells with micropipette aspiration. A computer vision tracking algorithm is developed for automatically measuring cell deformation parameters in real time $(30 \mathrm{~Hz})$ with a resolution down to 0.21 pixel, significantly enhancing the accuracy and efficiency of micropipette aspiration. To achieve a high characterization accuracy, the cell deformations and applied pressure changes are precisely synchronized using a data synchronization mechanism. Experimental results on both solid-like cells (interstitial cells) and liquid-like cells (neutrophils) quantitatively demonstrate that the visual tracking algorithm is capable of significantly increasing the efficiency and accuracy of micropipette aspiration. Among several characterized mechanical parameters, the viscoelastic properties of porcine aortic valve interstitial cells were, for the first time, quantified in this study.
\end{abstract}

Note to Practitioners-Micropipette aspiration is a widely used technique for characterizing mechanical properties of individual biological cells. Automated cell contour measurements can prove very useful for users of micropipette aspiration to avoid hours of laborious postprocessing, which is necessary with existing systems. Furthermore, compared with manual measurements, sub-pixel tracking of cell deformation parameters leads to a higher accuracy in quantifying cell mechanical properties.

Index Terms-Cell contour visual tracking, high accuracy, micropipette aspiration, precise data synchronization.

\section{INTRODUCTION}

$\mathbf{M}$ ECHANICAL signals (i.e., applied forces and stresses) are capable of regulating the phenotypic expression of biological cells. These mechanical stimuli are particularly important for highly mechanically responsive cells such as cardiovascular, musculoskeletal, and bone cells. For example, it is known that mechanical forces play a major role in the regulation of cell adhesion and cytoskeletal organization [1]. While it

Manuscript received October 15, 2007; revised March 12, 2008. First published June 02, 2009; current version published July 01, 2009. This paper was recommended for publication by Associate Editor K. Bohringer and Editor D. Meldrum upon evaluation of the reviewers' comments. This research was supported in part by the Natural Sciences and Engineering Research Council of Canada through a Discovery Grant and in part by the Ontario Ministry of Research and Innovation through an Early Researcher Award.

X. Liu and Y. Sun are with the Advanced Micro and Nanosystems Laboratory, University of Toronto, Toronto, ON M5S 3G8, Canada (e-mail: sun@mie. utoronto.ca).

Y. Wang is with the Schulich School of Medicine and Dentistry, University of Western Ontario, London, ON N6A 5C1, Canada.

Color versions of one or more of the figures in this paper are available online at http://ieeexplore.ieee.org.

Digital Object Identifier 10.1109/TASE.2009.2021356

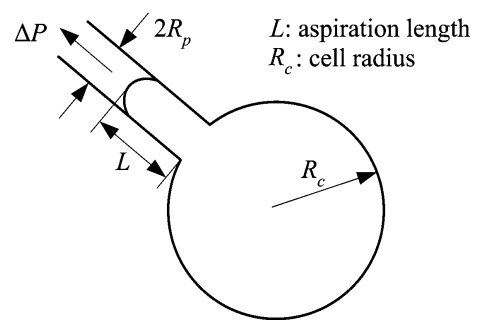

Fig. 1. Schematic of micropipette aspiration.

is important to investigate how mechanical forces/stresses regulate cell responses, it is also significant to characterize the mechanical properties of individual cells for understanding cellular structures and functions, predicting their response to mechanical stimuli, and correlating mechanical properties to disease states [2].

For measuring mechanical properties of a living cell, the cell must be deformed in some way by a known force or stress and its deformations measured. Besides atomic force microscopy [3], laser trapping [4], magnetic bead measurements [5], and microelectromechanical systems (MEMS)-based force measurements [6], micropipette aspiration is a popular technique for quantifying mechanical properties of individual cells, such as Young's modulus and viscosity [7].

As shown in Fig. 1, the micropipette aspiration technique applies a sucking pressure $\Delta P$ to deform a cell, and a portion of the cell is elongated into the micropipette. Experimentally, biological cells can be divided into two types, solid-like cells and liquid-like cells, according to their different responses to sucking pressures [7]. Liquid-like cells (e.g., neutrophils [8], [9] and erythrocytes [10]) behave like a liquid droplet, and an applied pressure above a critical value causes complete cell aspiration into the micropipette. On the other hand, a cell exhibiting behavioral characteristics of a solid (e.g., endothelial cells [11], [12] and chondrocytes [13]) continues to enter a finite distance into the micropipette when applied pressure exceeds the critical value. For characterizing both solid-like and liquid-like cells, the applied negative pressure $(\Delta P)$ and resulting cell deformation parameters (e.g., aspiration length $L$ and cell contour radius outside micropipette $R_{c}$ ) must be experimentally measured. Various continuum models [8], [9], [11], [12], [14] are employed for quantifying cellular mechanical parameters.

Key experimental factors that determine the validity of mechanical characterization results include the accuracy of applied pressure, the accuracy of cell geometrical parameter measurements, and the synchronization of applied pressure and resulting geometrical changes of the cell. In conventional micropipette 


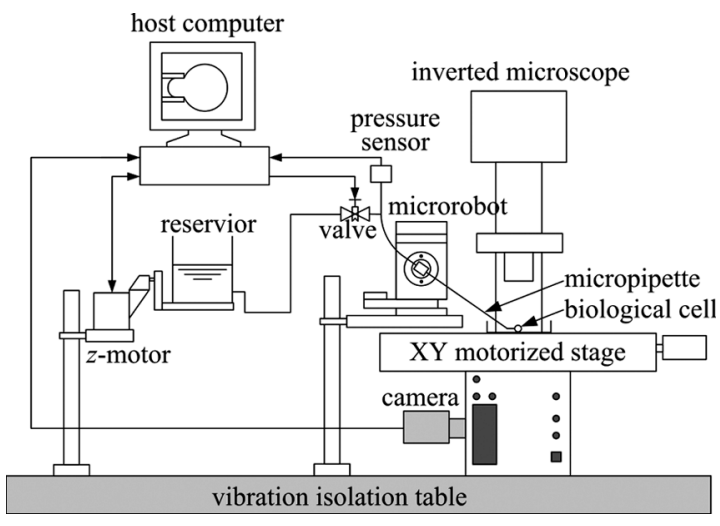

Fig. 2. Schematic diagram of the micropipette aspiration system.

systems, cell deformation images and pressure data are acquired separately using a video recorder and a pressure sensor, and the measured pressure data are recorded on the video tape to achieve data synchronization. Typically, a large number of images (30 frames/s for minutes) are recorded for postprocessing in which cell contours are manually measured with a cursor on the video screen. Cell contour measurements are tedious for human operators, making micropipette aspiration a time consuming technique. More importantly, offline manual measurements can produce significant errors in subsequent mechanics modeling due to the low accuracy of manual measurements. In the most ideal case, manual measurements are still limited to a resolution of one image pixel. Thus, the development of an automated computer vision tracking algorithm capable of conducting cell contour measurements in real time ( 30 frames/s) with a sub-pixel resolution, is highly desired.

Few attempts have been made by leveraging image processing and computer vision techniques to improve the accuracy and efficiency of the micropipette aspiration technique. In [15] and [16], algorithms were developed for offline postprocessing recorded images of aspirated lipid vesicles. These algorithms measure deformation parameters by locating only a few determinant points on a vesicle contour since the outside portion of an aspirated vesicle is largely spherical. However, these algorithms do not consider/accommodate the aspiration of solid-like cells (e.g., interstitial cells) that often reveal irregular contours, in which all points on an irregular cell contour should be accurately tracked in order to warrant a high measurement accuracy.

This paper presents a sub-pixel cell contour visual tracking algorithm, a precise data synchronization mechanism, and their application to real-time, high-accuracy micropipette aspiration. The images of deformed cell contours and sucking pressure data are precisely synchronized on a host computer. The visual tracking algorithm is capable of automatically locating irregular cell contours and micropipette tip, and therefore measuring the cell deformation parameters in real time during the aspiration process, enabling online characterization of cellular mechanical properties. Experiments demonstrate that the automated approach of cell contour measurements greatly improves the accuracy and efficiency in quantifying cellular mechanical parameters with the micropipette aspiration technique.

\section{SYSTEM SETUP}

The experimental system, schematically shown in Fig. 2, consists of an inverted microscope (Olympus IX81) with a CMOS camera (Basler A601f), a 3-DOF microrobot (Sutter MP-285) for positioning the micropipette, a XY motorized stage (Prior Scientific ProScan II) for positioning cell samples, a pressure system for generating well-controlled negative pressures, and a host computer with a data acquisition card (NI PCI-6229) for cell contour visual tracking and pressure data acquisition.

The sucking pressure unit includes an adjustable water reservoir, a $z$-motor, a solenoid valve, and a pressure sensor (All Sensors U.S.). Pressure is controlled by adjusting the height of the reservoir using the $z$-motor with a positioning resolution of $40 \mathrm{~nm}$. The pressure sensor is located close to the micropipette tip to minimize the pressure-transmission-caused time lag between pressure measurements and applied pressure, which was determined to be within $3 \mathrm{~ms}$ through fluidic finite element simulation. The differential pressure sensor is silicon based with a temperature compensation module and an output amplification circuit that is capable of directly providing $0 \sim 4 \mathrm{~V}$ output to the data acquisition card. The pressure measurement resolution is $0.1 \mathrm{~Pa}$.

The system setup is mounted on a vibration isolation table. The microscopy images and sucking pressure data in the system are directly acquired and processed by the host computer, enabling precise synchronization of cell deformations and sucking pressure. The lapse time between the acquisitions of the two types of signals is less than $5 \mathrm{~ms}$.

\section{Automated Cell Contour Measurements}

In order to accurately measure cell deformation parameters, a sub-pixel visual tracking algorithm is developed. The algorithm is described in this section for measuring two typical cell deformation parameters, the aspiration length $L$, and the cell contour radius outside micropipette $R_{c}$ (Fig. 1). However, the algorithm is also capable of accurately tracking irregular cell contours outside micropipette, from which other cell deformation parameters can be extracted for different cell mechanics models.

For the measurement of aspiration lengths $(L)$, the micropipette tip and the leading edge of the aspirated cell portion must be precisely located. For the measurement of cell outer radius $\left(R_{c}\right)$, the cell contour outside the micropipette is accurately tracked. In this study, an identification algorithm is developed to locate the micropipette tip, providing a reference position for the measurement of $L$. A canny edge detector with adaptive thresholding is employed to detect the cell contour outside the micropipette. A Kalman tracking algorithm is responsible for tracking the leading edge that projects into the micropipette. The current implementation assumes that the cell locates to the right of the micropipette; however, the algorithm can be readily modified to account for location differences. The micropipette was adjusted and well aligned horizontally. In the following description, all images are first smoothed by a low-pass Gaussian filter, and the resulting images are denoted by $I_{i}(x, y)$. 
(a)
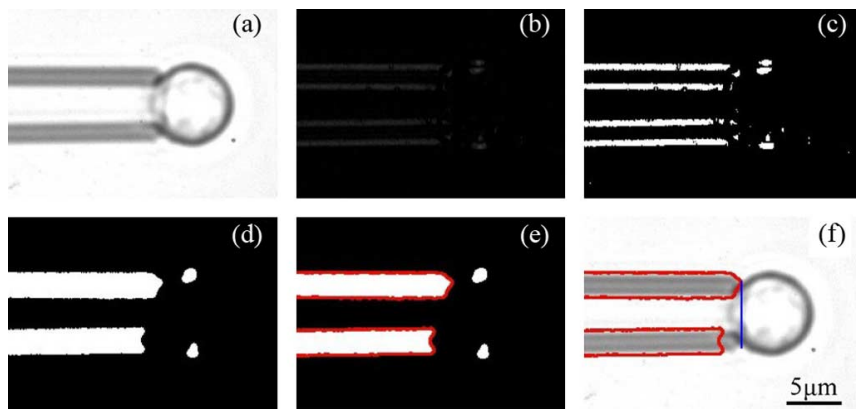

Fig. 3. Image sequence of micropipette tip identification. (a) Smoothed image of the first frame. (b) After gradient subtraction. (c) Adaptively thresholded. (d) Dilated image. (e) Top and bottom edges of micropipette walls found in (d). (f) Identified tip location.

\section{A. Micropipette Tip Identification}

In order to measure the length of cell projection into the micropipette, an identification algorithm is developed to locate the micropipette tip in the first frame of image where the establishment of initial contact between the cell and the side wall of the micropipette tip occurs. This contact blurs the vertical edge of the side wall. The edge of the cell in the proximity of the micropipette tip makes it difficult to precisely locate the micropipette tip using conventional edge detection algorithms, such as the Canny edge detector.

1) Gradient Subtraction: In our study, most of the edge information from the cell in the very first image frame is first removed using a gradient subtraction method. The absolute horizontal gradient is subtracted from the absolute vertical gradient in the first image frame. The resulting image [Fig. 3(b)] is

$$
D_{1}(x, y)=\left|\nabla_{y} I_{1}(x, y)\right|-\left|\nabla_{x} I_{1}(x, y)\right|
$$

2) Adaptive Thresholding : $D_{1}(x, y)$ is adaptively thresholded and converted into a binary image $B_{1}(x, y)$ [Fig. 3(c)] using the Otsu method [17].

3) Morphological Operation: A morphological close operation is used to connect the outer edge and inner edge of each micropipette wall into a single contour [Fig. 3(d)].

4) Tip Identification: By finding the two contours that span the greatest horizontal distance in the binary image Fig. 3(d), the top and bottom edges of the micropipette walls are found [Fig. 3(e)]. The horizontal image coordinate of the rightmost point in these two contours is identified as the location of the micropipette tip [Fig. 3(f)].

The identified micropipette tip is then used as a reference position for tracking cell deformations, $L$ and $R_{c}$. The processing time for tip identification is approximately $100 \mathrm{~ms}$. In our experiments, besides the use of a vibration-isolation table to minimize vibration-induced position change of the micropipette tip, other conditions (e.g., air flow) were also controlled to keep the micropipette tip location unchanged during the subsequent cell aspiration process. Thus, the micropipette tip identification is conducted only once, and does not sacrifice the real-time performance of cell contour tracking.

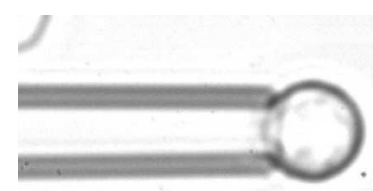

(a)
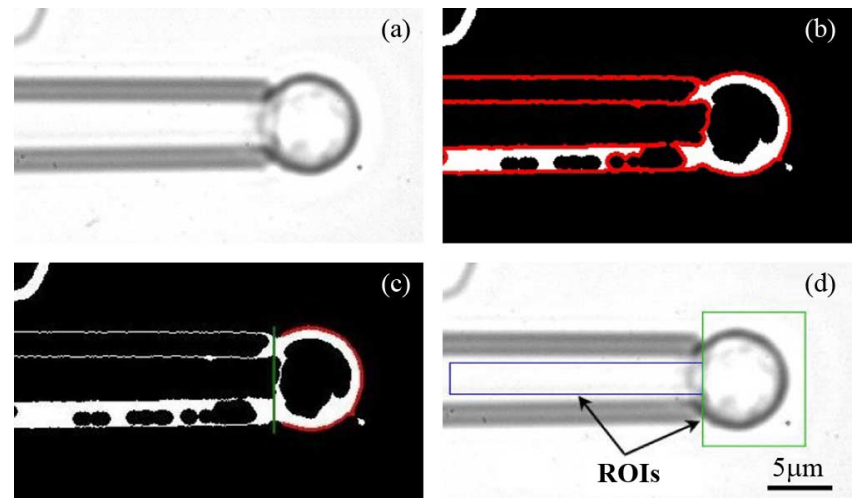

Fig. 4. Image sequence of tracking cell contour outside micropipette. (a) Smoothed image. (b) After adaptively thresholded Canny edge detection. (c) Cell contour determination using tip location information. (d) Determination of ROIs for subsequent tracking.

\section{B. Tracking of Cell Contour Outside Micropipette}

1) Canny Edge Detector With Adaptive Thresholding: The Canny edge detector tracks local maxima of an image gradient and eliminates weak edges by hysteresis thresholding using two constant threshold values. The high threshold $T_{h}$ is used to detect strong edges, while the low threshold $T_{l}$ detects weaker edges that are connected to strong edges. Due to the fact that different cell types reveal different image features, instead of frequently adjusting these two constant threshold values to accommodate different cell types, an adaptive thresholding algorithm for Canny edge detection [18] is employed for tracking the cell contour outside the micropipette.

In the adaptive thresholding method, the histogram $H(i)$ from the gradient magnitude of the image $D_{1}(x, y)$ is first calculated, and the maximum value $H_{\max }$ is determined. The deviation of $H(i)$ from $H_{\max }$ is given by

$$
\sigma=\sqrt{\frac{\sum_{i=0}^{N}\left[H(i)-H_{\max }\right]^{2}}{N}} .
$$

Accordingly, $T_{h}$ is set to be $H_{\max }+\sigma$. The deviation $\sigma^{\prime}$ of $H(i)$ from $H_{\max }$ is recalculated to exclude those pixels with gradient magnitudes above $T_{h}$. Finally, the low threshold value is determined as $T_{l}=H_{\max }+\sigma^{\prime}$. Fig. 4(b) shows the result after Canny edge detection with adaptively determined threshold values and a morphological close operation that is used to connect the Canny detected edges of the cell contour and micropipette walls into a single contour (shown in red).

2) Radius Measurement: After edge detection and close operation, the cell contour outside the micropipette [Fig. 4(c)] is extracted from the edge of the cell contour and micropipette walls [Fig. 4(b)] using the location information of the micropipette tip. In order to accurately determine the radius of the cell portion outside the micropipette $R_{c}$, a least square circle fitting algorithm [19] is used to fit the extracted cell contour into a circle for measurements. Note that the located cell contour data can also be used to extract other parameters required as input by different cell mechanics models (e.g., unit vectors normal to the cell contour [14]). 


\section{Tracking of Aspiration Lengths Into Micropipette}

Once the micropipette tip and the cell center are identified in the first frame of image, two ROIs, shown in Fig. 4(d), are determined and set for the subsequent tracking process. One ROI, to the right of the micropipette tip, is set to contain the cell portion outside the micropipette. The other ROI is to the left of the micropipette tip and contains the interior of the micropipette. The two ROIs are both symmetrical along the horizontal axis crossing the detected cell center.

The leading edge projecting into the micropipette must be accurately tracked in order to obtain accurate measurements of aspiration lengths $L$. However, the aspirated portion usually does not have a very distinct border, appearing rather faint. Active contour algorithms, such as Snakes, were found incapable of providing robust tracking performance due to the difficulty of controlling the snake's potential field to locate the leading edge. Snakes are particularly susceptible to the noise in the micropipette and the intracellular features that are often more prominent than the leading edge.

A Kalman tracking algorithm is developed that is capable of detecting weak edges and is robust to noises. The algorithm first obtains a horizontal gradient magnitude from the ROI that contains the leading edge. A low threshold is applied to the gradient for detecting the weak leading edge and producing a binary image. For example, a value of 20 was effective for human neutrophils in our experiments. All contours in the binary image are detected into chain codes, and the area for each contour is calculated. The leading edge is determined by selecting the leftmost contour with an area above a threshold value that is set proportional to the inner diameter of the micropipette, by which most of the noisy contours are excluded. The horizontal coordinate of the leftmost point in the leading edge is regarded as the preliminarily measured position. To further improve tracking robustness, this measured position is processed by a Kalman filter [20] to predict the real location of the leading edge and eliminate disturbances from the noisy points.

Briefly, the Kalman filter uses a state vector $\mathbf{X}_{i}=$ $\left[x_{i}, x_{i-1}, x_{i-2}\right]^{T}$ that consists of the estimated real positions of the leading edge in the past three images. A transition matrix is used to model the dynamics of the edge with the assumption of constant acceleration

$$
A=\left[\begin{array}{ccc}
3 & -3 & 1 \\
1 & 0 & 0 \\
0 & 1 & 0
\end{array}\right]
$$

The prediction step of the Kalman cycle is given by

$$
\mathbf{X}_{i}^{\prime}=\mathbf{A X}_{i-1}
$$

where $\mathbf{X}_{i}^{\prime}$ is the predicted state vector. Updating is conducted according to

$$
\mathbf{X}_{i}=\mathbf{X}_{i}^{\prime}+\mathbf{K}_{i}\left(z_{i}-\mathbf{H X}_{i}^{\prime}\right)
$$

where $\mathbf{K}_{i}$ is the Kalman gain [20], $z_{i}$ is the measured position of the leading edge, and $\mathbf{H}=\left[\begin{array}{lll}1 & 0 & 0\end{array}\right]$. The real position of the leading edge of the aspirated cell is determined by

$$
z_{i}^{\prime}=\mathbf{H X}_{i}
$$

\section{Cell Isolation AND Culture}

The micropipette aspiration system and the sub-pixel cell contour visual tracking algorithm were applied to characterizing mechanical properties of solid-like porcine aortic valve interstitial cells (PAVIC) and liquid-like human neutrophils.

Aortic valve leaflets were harvested from hog hearts $(\sim 7$ months old) obtained at a local abattoir. After rinsing with antibiotics, each leaflet was treated with collagenase $(150 \mathrm{U} / \mathrm{mL}$, $37^{\circ} \mathrm{C}, 20 \mathrm{~min}$ ) and the leaflet surfaces were scraped to remove endothelial cells. The leaflets were then minced, and digested with collagenase $\left(150 \mathrm{U} / \mathrm{mL}, 37{ }^{\circ} \mathrm{C}, 2 \mathrm{~h}\right)$. The supernatant was strained and centrifuged, and the cell pellet was resuspended in standard tissue culture medium (DMEM supplemented with $10 \%$ FBS and $1 \%$ antibiotics). The PAVICs were plated at $10^{4}$ cells $/ \mathrm{cm}^{2}$ on tissue culture flasks, and were kept in an incubator. The medium was changed every 2 days, and the cells were passaged when confluent. P2 cells were trypsinized and resuspended in standard tissue culture medium at $10^{5}$ cells $/ \mathrm{mL}$ for the experiments.

Human neutrophils used in the experiments were isolated from $5 \mathrm{ml}$ of blood from a healthy male volunteer (27 years old) using one-step Polymorphprep (Accurate Chemical, Westbury, NY) and were suspended in Hanks' balanced salt solution (HBSS) with $10 \%$ compatible plasma at $10^{5}$ cells $/ \mathrm{mL}$ for the experiments.

\section{EXPERIMENTAL RESULTS}

Both elastic and viscoelastic properties of PAVICs and human neutrophils were experimentally quantified. The experiments were conducted at room temperature of $22{ }^{\circ} \mathrm{C}$. Both types of cells were characterized between 15 and 90 min after passage/ isolation, and the results revealed no significant dependence on testing time during this period.

A glass slide (Fisher Scientific U.S.), coated with 1\% agar, was used as the substrate. Customized micropipettes (Humagen U.S.) used in the experiments were coated with $1 \%$ agar to avoid cell adhesion. A $60 \times$ objective (NA 0.7 ) plus a $1.6 \times$ coupler, and bright-field imaging were used for observing the cells. The calibrated pixel size is $0.11 \mu \mathrm{m} \times 0.11 \mu \mathrm{m}$.

Images and pressure data were acquired at a frequency of $30 \mathrm{~Hz}$. The cell contour visual tracking algorithm cost $10.2 \mathrm{~ms}$ for processing each frame of image, proving the real-time capability for measuring cell deformation parameters. For PAVICs, the tracking resolution of $L$ is 0.21 pixel. For human neutrophils, the tracking resolution is 0.29 pixel for $L$ and 0.42 pixel for $R_{c}$.

\section{A. Characterization of PAVICs}

1) Measurements of Young's Modulus: A total of ten PAVICs were tested in the experiments (five for elastic characterization and five for viscoelastic characterization). The average diameter of the PAVICs is $14.2 \mu \mathrm{m}$, and the inner diameter of the micropipettes for PAVIC characterization is $4.6 \mu \mathrm{m}$. An initial tare pressure $(\sim 40 \mathrm{~Pa})$ was applied for $60 \mathrm{~s}$ to the cell to form a seal between the micropipette and the cell [Fig. 5(a)]. Sucking pressure in 6-8 increasing steps from 0.04 to $1 \mathrm{kPa}$ was then applied to deform the cell. An interval of $60 \mathrm{~s}$ between two adjacent steps allowed the cell to reach equilibrium. Fig. 5 shows 

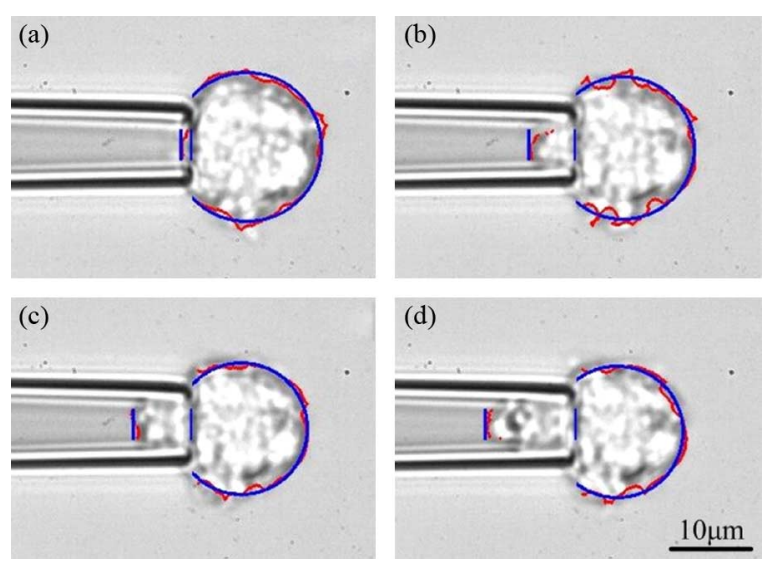

Fig. 5. Micropipette aspiration of a porcine aortic valve interstitial cell.

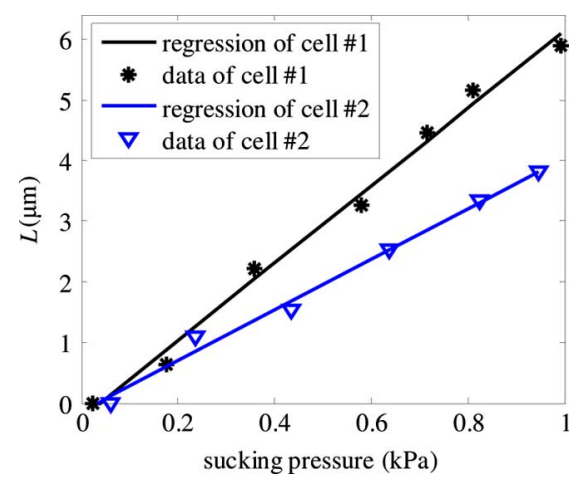

Fig. 6. PAVIC aspiration length versus sucking pressure.

the procedure of aspirating a PAVIC and the visual tracking results, where the tracked cell contours outside micropipette are highlighted by red lines.

The aspiration lengths $(L)$ were online measured by the subpixel cell contour tracking algorithm. Two representative sets of tracking data $(L)$ vs. sucking pressure are shown in Fig. 6. Homogenous half-space elastic model [11] for solid-like cells was employed for extracting Young's modulus values from the experimental data

$$
\Delta P=\frac{2 \pi}{3 \phi} E \frac{L}{R_{p}}
$$

where $E$ is the Young's modulus of the homogeneous solid, $\Delta P$ is the sucking pressure, $R_{p}$ is the inner diameter of the micropipette, and $\phi$ is a wall function determined by the pipette dimension and has a typical value of 2.1. The Young's modulus values of $345.8 \pm 142.5 \mathrm{~Pa}$ were obtained based on the measurements of five PAVICs. This result is comparable to the previously reported data of PAVICs $(\sim 449 \mathrm{~Pa})$ [21].

2) Measurements of Viscoelastic Properties: To date, there are no reported results on the viscoelastic properties of PAVICs, which are significant to quantify for understanding sclerosis (thickening and calcification) of the aortic valve. In this study, the viscoelastic properties of the PAVICs were quantified using the homogenous half-space linear viscoelastic model [12].

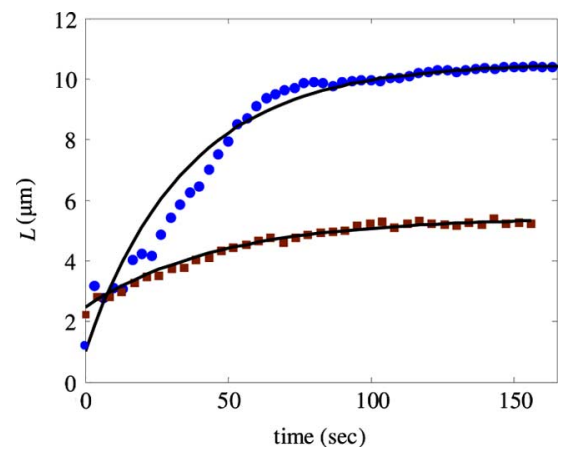

Fig. 7. Viscoelastic response of PAVICs.

TABLE I

PAVIC Viscoelastic PaRAMETERS (BASED ON Five Cells)

\begin{tabular}{|c|c|c|c|c|}
\hline Parameters & $k_{1}(\mathrm{~Pa})$ & $k_{2}(\mathrm{~Pa})$ & $\tau(\mathrm{s})$ & $\mu(\mathrm{Pa} \cdot \mathrm{s})$ \\
\hline Values & $153.8 \pm 59.9$ & $310.9 \pm 70.4$ & $45.2 \pm 11.6$ & $4330.5 \pm 958.1$ \\
\hline
\end{tabular}

When a stepwise sucking pressure is applied to the cell, cell deformations are

$$
L(t)=L_{\infty}\left[1-\frac{k_{2}}{k_{1}+k_{2}} \exp \left(-\frac{t}{\tau}\right)\right] h(t)
$$

where

$$
L_{\infty}=\lim _{t \rightarrow \infty} L(t)=\frac{2 R_{p} \Delta P}{\pi k_{1}}
$$

$h(t)$ is a unit step function

$$
h(t)= \begin{cases}1, & t>0 \\ 0, & t \leq 0\end{cases}
$$

$k_{1}$ and $k_{2}$ are the elastic constants in the Kelvin model, and $\tau$ is a time constant given by

$$
\tau=\frac{\mu}{k_{1}}\left(1+\frac{k_{1}}{k_{2}}\right)
$$

where $\mu$ is the coefficient of viscosity in the Kelvin model. From (7), the initial aspiration length $\left(L_{0+}\right)$ due to the elastic effect can be derived as

$$
L_{0+}=\frac{k_{1}}{k_{1}+k_{2}} L_{\infty}
$$

$k_{1}$ is experimentally obtained by measuring the asymptotic length of the elongated portion $L_{\infty} . k_{2}$ is experimentally obtained by measuring $L_{\infty}$ and $L_{0+}$. Fitting the experimental data into (7) determines $\tau$ and $\mu$.

In the experiments, a sucking pressure step of $860 \mathrm{~Pa}$ (standard deviation $\pm 56.7 \mathrm{~Pa}$ ) was applied to the PAVICs, while resulting aspiration lengths $L$ were measured in real time. The initial elastic response $L_{0+}$, was precisely measured, and a high accuracy is warranted by precise synchronization in the system. The experimental data were fitted into (7), and the parameters of $k_{1}, k_{2}, \tau$, and $\mu$ in the model were computed according to (8), (10), and (11). Fig. 7 shows two representative sets of tracking results of $L$ and the fitted curves. The statistics of the five aspirated PAVICs for viscoelastic characterization are summarized in Table I. 


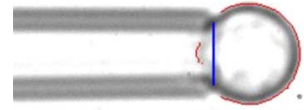

(a)

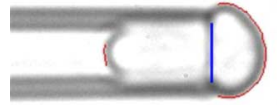

(c)

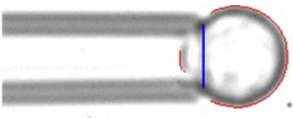

(b)

(d) $\quad \underline{5 \mu \mathrm{m}}$

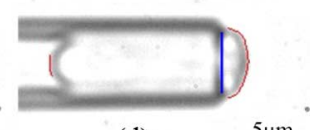

Fig. 8. Micropipette aspiration of a human neutrophil. (a) Initial state after the establishment of a seal. (b) The critical state. (c) Deformed cell flowing halfway into micropipette. (d) Cell completely flowing into micropipette except the hemispherical portion.

Compared with the reported characterization results of porcine aortic endothelial cells [12], which are another type of solid-like cells, the elastic constants of the PAVICs, $k_{1}$ and $k_{2}$, are approximately twice those of the endothelial cells, and the viscosity coefficient of the PAVICs, $\mu$, is about half of the value of the endothelial cells. These results indicate that the PAVICs have a higher stiffness and a lower viscosity than porcine aortic endothelial cells.

\section{B. Characterization of Human Neutrophils}

Fig. 8 shows the procedure of aspirating a neutrophil and the tracking results. The inner diameter of the micropipettes used in the neutrophil experiments is $4 \mu \mathrm{m}$. The average diameter of the neutrophils is $8.4 \mu \mathrm{m}$. The mechanical parameters of human neutrophils were determined through cortical shell-liquid core models [8], [9].

At the beginning of aspiration, the sucking pressure was controlled to increase until the aspiration length was equal to the micropipette radius, which was measured on line by the cell contour visual tracking algorithm. The critical pressure and the radius $R_{c}$ at this point were automatically recorded by the system, and the cortical tension of the surrounding shell can be achieved by the equilibrium equation [8], [9]

$\Delta P=2 T\left(\frac{1}{R_{p}}-\frac{1}{R_{c}}\right),\left(\Delta P=\Delta P_{c}\right.$ when $\left.\frac{L_{p}}{R_{p}}=1\right)$

where $T$ is the cortical tension, $R_{c}$ is the radius of the cell portion outside the pipette, and $\Delta P_{c}$ is the critical pressure. After this critical point, the sucking pressure was increased by a step to $820 \mathrm{~Pa}$ (standard deviation $\pm 48.8 \mathrm{~Pa}$ ), causing the cell to automatically flow into the micropipette. During this process, the aspiration lengths and the radius of the cell portion outside the micropipette were measured in real time. Finally, the measured parameters were fitted into the following equation to calculate the viscosity of the cytoplasm [9]

$$
\eta=\frac{R_{p} \Delta P}{\left(\frac{d L}{d t}\right) m\left(\frac{1-R_{p}}{R_{c m}}\right)}
$$

where $m$ is a constant with a typical value of 6 , and $R_{c m}$ is the radius of the outer cell portion corresponding to the middle point in the linear region of $L$ versus time in experimental data.

Fig. 9 shows the tracking results of aspiration lengths $L$ for five tested neutrophils. The linear regions of these curves were fitted to calculate the constant flowing velocities. The measured flowing velocities and the stepwise sucking pressure

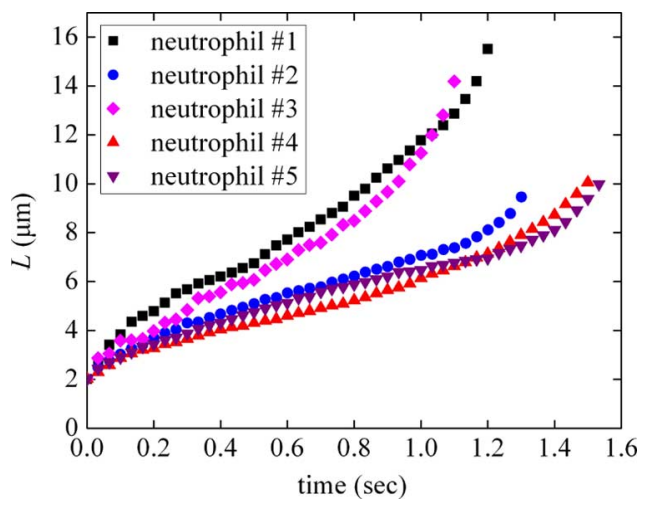

Fig. 9. Neutrophil tracking results of aspiration lengths into micropipette.

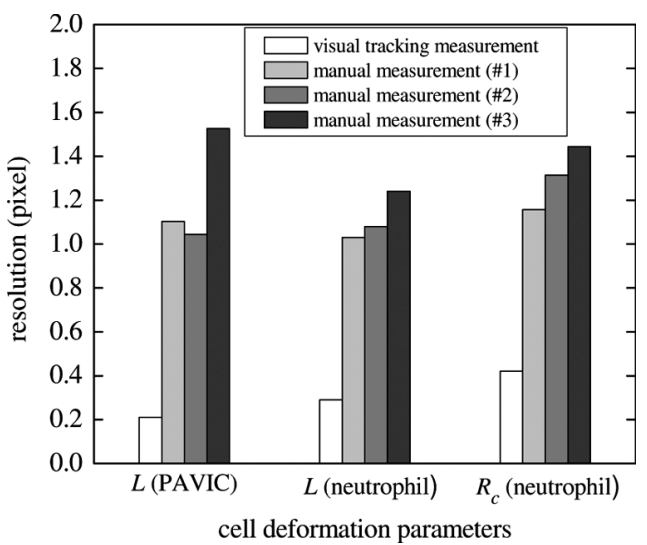

Fig. 10. Measurement resolutions achieved by human operators and by automated cell contour visual tracking.

TABLE II

Average Time for Manually MEASURING OnE Frame of IMAgE

\begin{tabular}{|c|c|c|}
\hline Cell types & PAVIC & Neutrophil \\
\hline Human operator \#1 & $25.2 \mathrm{sec}$ & $30.4 \mathrm{sec}$ \\
\hline Human operator \#2 & $19.1 \mathrm{sec}$ & $32.9 \mathrm{sec}$ \\
\hline Human operator \#3 & $10.9 \mathrm{sec}$ & $20.9 \mathrm{sec}$ \\
\hline
\end{tabular}

$(804 \pm 48.3 \mathrm{~Pa})$ were finally substituted into (13) for determining viscosities. For the five tested neutrophils, viscosity is $191.9 \pm 86.1 \mathrm{~Pa} \cdot \mathrm{s}$, and the determined cortical tension is $40.9 \pm 10.8 \mathrm{pN} / \mu \mathrm{m}$, which are comparable with previous results $(\sim 135 \mathrm{~Pa} \cdot \mathrm{s}$ for viscosity and $\sim 35 \mathrm{pN} / \mu \mathrm{m}$ for cortical tension) reported in [8] and [9].

\section{DISCUSSION}

Images of the 15 characterized cells (ten PAVICs and five neutrophils) at aspiration equilibrium were measured by three welltrained human operators. Manual measurement resolutions were quantified by calculating the standard deviation of manually measured data for each cell deformation parameter (i.e., $L$ and $\left.R_{c}\right)$. Fig. 10 summarizes averaged resolutions of manual measurements together with automated visual tracking resolutions.

The much better measurement resolutions of automated cell contour visual tracking permit more accurate measurements of cell deformation parameters. Furthermore, the average time required for processing each frame of image in manual measurements is listed in Table II. Compared to the speed of manual measurements (10.9 s-32.9 s/image), automated 
TABLE III

ERRors of Cell Parameter QuANTIFICATION In MANUAL MEASUREMENTS

\begin{tabular}{|c|c|c|c|c|}
\hline \multicolumn{2}{|c|}{$\begin{array}{l}\text { Mechanica } \\
\text { parameters }\end{array}$} & $\begin{array}{c}\text { Human } \\
\text { operator \#1 }\end{array}$ & $\begin{array}{c}\text { Human } \\
\text { operator \#2 }\end{array}$ & $\begin{array}{c}\text { Human } \\
\text { operator \#3 }\end{array}$ \\
\hline \multirow{5}{*}{ 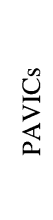 } & $E$ & $23.2 \%$ & $19.8 \%$ & $35.7 \%$ \\
\hline & $k_{1}$ & $16.9 \%$ & $11.5 \%$ & $14.3 \%$ \\
\hline & $k_{2}$ & $39.2 \%$ & $45.6 \%$ & $40.1 \%$ \\
\hline & $\tau$ & $20.1 \%$ & $12.6 \%$ & $26.7 \%$ \\
\hline & $\mu$ & $36.6 \%$ & $29.3 \%$ & $21.2 \%$ \\
\hline \multirow{2}{*}{ 总 } & $T_{c}$ & $41.3 \%$ & $32.5 \%$ & $50.6 \%$ \\
\hline & $\eta$ & $13.0 \%$ & $21.2 \%$ & $27.1 \%$ \\
\hline
\end{tabular}

visual tracking provides a much higher measurement efficiency (10.2 ms/image). Table III summarizes mechanical parameter quantification errors of manual measurements, where each entry represents the average value from five cells. One can see that the sub-pixel visual tracking algorithm is capable of improving the measurement accuracy by up to $50.6 \%$.

The cell contours measured in the micropipette aspiration experiments are only 2-D cross-sectional contours that do not reveal the complete 3-D cell geometry. Consequently, errors could exist in the absolute cell geometrical parameters (e.g., cell volume) measured using the micropipette aspiration technique. Further enhancement of the measurement accuracy of such absolute cell geometrical parameters can possibly be achieved by detecting 3-D cell geometries via focus-based 3-D reconstruction algorithms [22], [23].

\section{CONCLUSION}

This paper presented a real-time, sub-pixel cell contour measurement technique with a resolution down to 0.21 pixel and a precise data synchronization mechanism for real-time, highaccuracy micropipette aspiration. The cell contour tracking algorithm and the experimental system were applied to conduct micropipette aspiration experiments on porcine aortic valve interstitial cells and human neutrophils, demonstrating a $50.6 \%$ improvement of characterization accuracy over manual measurements. These automation techniques will prove important for realizing highly efficient, accurate characterization of mechanical properties of biological cells using micropipette aspiration.

\section{ACKNOWLEDGMENT}

The authors thank Prof. C. Simmons' group for assistance with interstitial cell preparation, and Prof. G. Downey and Prof. M. Glogauer for the assistance with human neutrophils preparations.

\section{REFERENCES}

[1] C. S. Chen, M. Mrksich, S. Huang, G. M. Whitesides, and D. E. Ingber, "Geometric control of cell life and death," Science, vol. 276, pp. $1425-1428,1997$.

[2] G. Y. H. Lee and C. T. Lim, "Biomechanics approaches to studying human diseases," Trends Biotech., vol. 25, pp. 111-118, 2007.
[3] E. K. Dimitriadis, F. Horkay, J. Maresca, B. Kachar, and R. S. Chadwick, "Determination of elastic moduli of thin layers of soft material using the atomic force microscope," Biophys. J., vol. 82, pp. 2798-2810, 2002.

[4] J. W. Dai and M. P. Sheetz, "Mechanical properties of neuronal growth cone membranes studied by tether formation with laser optimal tweezers," Biophys. J., vol. 68, pp. 988-996, 1995.

[5] A. R. Bausch, F. Ziemann, A. A. Boulbitch, K. Jacobson, and E. Sachmann, "Local measurements of viscoelastic parameters of adherent cell surfaces by magnetic bead microrheometry," Biophys. J., vol. 75, pp. 2038-2049, 1998.

[6] Y. Sun, K. T. Wan, B. J. Nelson, J. Bischof, and K. Roberts, "Mechanical property characterization of the mouse zona pellucida," IEEE Trans. NanoBioSci., vol. 2, pp. 279-286, 2003.

[7] R. M. Hochmuth, "Micropipette aspiration of living cells," J. Biomech., vol. 33, pp. 15-22, 2000.

[8] E. Evans and A. Yeung, "Apparent viscosity and cortical tension of blood granulocytes determined by micropipette aspiration," Biophys. J., vol. 56, pp. 151-160, 1989.

[9] D. Needham and R. M. Hochmuth, "Rapid flow of passive neutrophils into a $4 \mu \mathrm{m}$ pipet and measurement of cytoplasmic viscosity," $J$. Biomech. Eng., vol. 112, pp. 269-276, 1990.

[10] D. E. Discher, D. H. Boal, and S. K. Boey, "Simulations of the erythrocyte cytoskeleton at large deformation II: Micropipette aspiration," Biophys. J., vol. 75, pp. 1584-1597, 1998.

[11] D. P. Theret, M. J. Levesque, M. Sato, R. M. Nerem, and L. T. Wheeler, "The application of a homogeneous half-space model in the analysis of endothelial cell micropipette measurements," Trans. ASME, vol. 110, pp. 190-199, 1988.

[12] M. Sato, D. P. Theret, L. T. Wheeler, N. Ohshima, and R. M. Nerem, "Application of the micropipette technique to the measurement of cultured porcine aortic endothelial cell viscoelastic properties," $J$. Biomech. Eng., vol. 112, pp. 263-268, 1990.

[13] W. R. Jones, H. P. T. Beall, G. M. Lee, S. S. Kelley, R. M. Hochmuth, and F. Guilak, "Alterations in the Young's modulus and volumetric properties of chondrocytes isolated from normal and osteoarthritic human cartilage," J. Biomech., vol. 32, pp. 119-127, 1999.

[14] M. Herant, W. A. Marganski, and M. Dembo, "The mechanics of neutrophils: Synthetic modeling of three experiments," Biophys. J., vol. 84, pp. 3389-3413, 2003.

[15] V. Heinrich and W. Rawicz, "Automated, high-resolution micropipet aspiration reveals new insight into the physical properties of fluid membranes," Langmuir, vol. 21, pp. 1962-1971, 2005.

[16] J. R. Henriksen and J. H. Ipsen, "Measurement of membrane elasticity by micro-pipette aspiration," Eur. Phys. J. E, vol. 14, pp. 149-167, 2004.

[17] N. Otsu, "A threshold selection method from gray-level histograms," IEEE Trans. Syst. Man. Cybern., vol. 9, pp. 62-66, 1979.

[18] W. Zhi, Q. Li, S. Zhong, and S. He, "Fast adaptive threshold for the canny edge detector," Proc. SPIE, vol. 6044, p. 60441Q, 2005.

[19] X. Y. Liu, Y. Sun, W. H. Wang, and B. M. Lansdorp, "Vision-based cellular force measurement using an elastic microfabricated device," $J$. Micromech. Microeng., vol. 17, pp. 1281-1288, 2007.

[20] P. Zarchan and H. Musoff, Fundamentals of Kalman Filtering: A Practical Approach. Reston, VA: AIAA Publications, 2005.

[21] W. D. Merryman, I. Youn, H. D. Lukoff, P. M. Krueger, F. Guilak, R. A. Hopkins, and M. S. Sacks, "Correlation between heart valve interstitial cell stiffness and transvalvular pressure: Implications for collagen biosynthesis," Amer. J. Physiol. Heart Circ. Physiol., vol. 290, pp. 224-231, 2005.

[22] J. Ens and P. Lawrence, "An investigation of methods for determining depth from focus," IEEE Trans. Pattern Anal. Mach. Intell., vol. 15, pp. 97-108, 1993.

[23] S. K. Nayar and Y. Nakagawa, "Shape from focus," IEEE Trans. Pattern Anal. Mach. Intell., vol. 16, pp. 824-831, 1994.

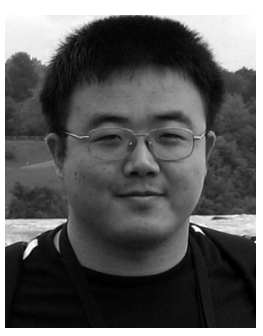

Xinyu Liu (S'06) received the B.S. and M.S. degrees in mechanical engineering from the Harbin Institute of Technology, Harbin, China, in 2002 and 2004, respectively. He is currently working towards the Ph.D. degree in the Department of Mechanical and Industrial Engineering, University of Toronto, Toronto, ON, Canada.

His research area is microrobotics and MEMS assisted manipulation of biomaterials. 


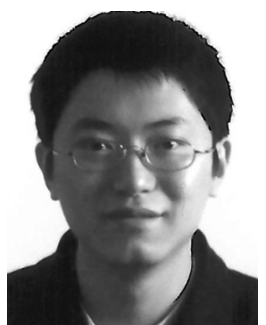

Yifei Wang (S'06) received the B.A.Sc. degree in electrical engineering from the University of Toronto, Toronto, ON, Canada, in 2007. He is currently working towards the M.D. degree at the Schulich School of Medicine and Dentistry, University of Western Ontario, London, ON, Canada.

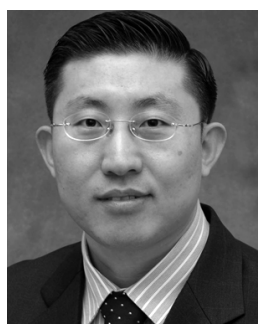

Yu Sun (S'01-M'03-SM'07) received the B.S. degree in electrical engineering from the Dalian University of Technology, Dalian, China, in 1996, the M.S. degree from the Institute of Automation, Chinese Academy of Sciences, Beijing, China, in 1999, the M.S. degree in electrical engineering and the $\mathrm{Ph} . \mathrm{D}$. degree in mechanical engineering from the University of Minnesota, Minneapolis, in 2001 and 2003, respectively.

He held a Research Scientist position at the Swiss Federal Institute of Technology (ETH-Zürich) before joining the Faculty of the University of Toronto, where he is an Assistant Professor of Mechanical and Industrial Engineering and is jointly appointed at the Institute of Biomaterials and Biomedical Engineering and the Electrical and Computer Engineering Department. He established and directs the Advanced Micro and Nanosystems Laboratory at Toronto and is the Canada Research Chair in Micro and Nano Engineering Systems. His research areas are design and fabrication of M/NEMS devices and micronanorobotic manipulation of biomaterials and nanomaterials.

Dr. Sun is a recipient of the MRI Innovations Award, the Premier's Early Researcher Award for Research in "MEMS-Assisted Micro and Nanomanipulation of Biological Cells and Nanomaterials," and the Best Paper Award at the 2007 IEEE International Conference on Automation Science and Engineering. He is an Associate Editor of the IEEE TRANSACTIONS ON AUTOMATION SCIENCE AND ENGINEERING. 